\title{
On the non-Lipschitz stochastic differential equations driven by fractional Brownian motion
}

Bin Pei and Yong $\mathrm{Xu}^{*}$

\section{"Correspondence:}

hsux3@nwpu.edu.cn

Department of Applied

Mathematics, Northwestern

Polytechnical University, Xi'an,

710072, China

\section{包 Springer}

\begin{abstract}
In this paper, we use a successive approximation method to prove the existence and uniqueness theorems of solutions to non-Lipschitz stochastic differential equations (SDEs) driven by fractional Brownian motion (fBm) with the Hurst parameter $H \in\left(\frac{1}{2}, 1\right)$. The non-Lipschitz condition which is motivated by a wider range of applications is much weaker than the Lipschitz one. Due to the fact that the stochastic integral with respect to $\mathrm{fBm}$ is no longer a martingale, we definitely lost good inequalities such as the Burkholder-Davis-Gundy inequality which is crucial for SDEs driven by Brownian motion. This point motivates us to carry out the present study.
\end{abstract}

Keywords: fractional Brownian motion; existence and uniqueness; stochastic differential equations; non-Lipschitz condition

\section{Introduction}

Stochastic differential equations (SDEs) have been greatly developed and are well known to model diverse phenomena, including but not limited to fluctuating stock prices, physical systems subject to thermal fluctuations, forecasting the growth of a population, from various points of view [1-4]. There is no doubt that the mathematical models under a random disturbance of 'Gaussian white noise' have seen rapid development. However, it is not appropriate to model some real situations where stochastic fluctuations with longrange dependence might exist. Due to the long-range dependence of the $\mathrm{ABm}$ which was introduced by Hurst [5], Kolmogorov [6], Mandelbrot [7] originally, SDEs driven by fBm have been used as the models of a number of practical problems in various fields, such as queueing theory, telecommunications, and economics [8-10].

On most occasions, the coefficients of SDEs driven by $\mathrm{fBm}$ are assumed to satisfy the Lipschitz condition. The existence and uniqueness of solutions of SDEs driven by fBm with Lipschitz condition have been studied by many scholars [11-14]. However, this Lipschitz condition seemed to be considerably strong when one discusses variable applications in real world. For example, the hybrid square root process and the one-dimensional semilinear SDEs with Markov switching. Such models appear widely in many branches of science, engineering, industry and finance [15-17]. Therefore, it is important to obtain some weaker condition than the Lipschitz one under which the SDEs still have unique solutions. Fortunately, many researchers have investigated the SDEs under non-Lipschitz condition

(c) 2016 Pei and Xu. This article is distributed under the terms of the Creative Commons Attribution 4.0 International License (http://creativecommons.org/licenses/by/4.0/), which permits unrestricted use, distribution, and reproduction in any medium, provided you give appropriate credit to the original author(s) and the source, provide a link to the Creative Commons license, and indicate if changes were made. 
and they presented many meaningful results [18-22]. But, to the best of our knowledge, the existence and uniqueness of solutions of SDEs driven by $\mathrm{fBm}$ with a non-Lipschitz condition have not been considered. Since $\mathrm{fBm}$ is neither a semi-martingale nor a Markov process, we definitely lost good inequalities such as the Burkholder-Davis-Gundy inequality, which is crucial for SDEs driven by Brownian motion. Then it seems not to be very easy to obtain the existence and uniqueness of solutions to non-Lipschitz SDEs with $\mathrm{fBm}$. This point motivates us to carry out the present study.

We in the present paper discuss the SDEs with $\mathrm{fBm}$ under the non-Lipschitz condition. Using the successive approximation method, the existence and uniqueness theorems of solutions to the following non-Lipschitz SDEs driven by $\mathrm{fBm}$ are proved:

$$
X(t)=X(0)+\int_{0}^{t} b(s, X(s)) d s+\int_{0}^{t} \sigma(s, X(s)) d B^{H}(s), \quad t \in[0, T]
$$

where the initial data $X(0)=\xi$ is a random variable, $0<T<\infty$, the process $B^{H}(t)$ represents the fBm with Hurst index $H \in\left(\frac{1}{2}, 1\right)$ defined in a complete probability space $(\Omega, \mathcal{F}, \mathbb{P})$, and $b(t, X(t)):[0, T] \times R \rightarrow R$ and $\sigma(t, X(t)):[0, T] \times R \rightarrow R$ are all measurable functions; $\int_{0}^{t} \cdot d B^{H}(s)$ stands for the stochastic integral with respect to $\mathrm{fBm}$.

\section{Preliminaries}

Let $(\Omega, \mathcal{F}, \mathbb{P})$ be a complete probability space. SDEs with respect to $\mathrm{fBm}$ have been interpreted via various stochastic integrals, such as the Wick integral, the Wiener integral, the Skorohod integral, and path-wise integrals [13,23-26]. In this paper, we consider the path-wise integrals [27] with respect to $\mathrm{fBm}$.

Let $\varphi: R_{+} \times R_{+} \rightarrow R_{+}$be defined by

$$
\varphi(t, s)=H(2 H-1)|t-s|^{2 H-2}, \quad t, s \in R_{+},
$$

where $H$ is a constant with $\frac{1}{2}<H<1$.

Let $g: R_{+} \rightarrow R$ be Borel measurable.

Define

$$
L_{\varphi}^{2}\left(R_{+}\right)=\left\{g:\|g\|_{\varphi}^{2}=\int_{R_{+}} \int_{R_{+}} g(t) g(s) \varphi(t, s) d s d t<\infty\right\} .
$$

If we equip $L_{\varphi}^{2}\left(R_{+}\right)$with the inner product

$$
\left\langle g_{1}, g_{2}\right\rangle_{\varphi}=\int_{R_{+}} \int_{R_{+}} g_{1}(t) g_{2}(s) \varphi(t, s) d s d t, \quad g_{1}, g_{2} \in L_{\varphi}^{2}\left(R_{+}\right)
$$

then $L_{\varphi}^{2}\left(R_{+}\right)$becomes a separable Hilbert space.

Let $\mathcal{S}$ be the set of smooth and cylindrical random variables of the form

$$
F(\omega)=f\left(\int_{0}^{T} \psi_{1}(t) d B_{t}^{H}, \ldots, \int_{0}^{T} \psi_{n}(t) d B_{t}^{H}\right)
$$

where $n \geq 1, f \in \mathcal{C}_{b}^{\infty}\left(R^{n}\right)$ (i.e. $f$ and all its partial derivatives are bounded), and $\psi_{i} \in \mathcal{H}$, $i=1,2, \ldots, n$. $\mathcal{H}$ is the completion of the measurable functions such that $\|\psi\|_{\varphi}^{2}<\infty$ and $\left\{\psi_{n}\right\}$ is a sequence in $\mathcal{H}$ such that $\left\langle\psi_{i}, \psi_{j}\right\rangle_{\varphi}=\delta_{i j}$. 
The Malliavin derivative $D_{t}^{H}$ of a smooth and cylindrical random variable $F \in \mathcal{S}$ is defined as the $\mathcal{H}$-valued random variable:

$$
D_{t}^{H} F=\sum_{i=1}^{n} \frac{\partial f}{\partial x_{i}}\left(\int_{0}^{T} \psi_{1}(t) d B_{t}^{H}, \ldots, \int_{0}^{T} \psi_{n}(t) d B_{t}^{H}\right) \psi_{i}(t) .
$$

Then, for any $p \geq 1$, the derivative operator $D_{t}^{H}$ is a closable operator from $L^{p}(\Omega)$ into $L^{p}(\Omega ; \mathcal{H})$. Next, we introduce the $\varphi$-derivative of $F$ :

$$
D_{t}^{\varphi} F=\int_{R_{+}} \varphi(t, v) D_{v}^{H} F d v
$$

The elements of $\mathcal{H}$ may not be functions but distributions of negative order. Thanks to this, it is convenient to introduce the space $|\mathcal{H}|$ of the measurable function $h$ on $[0, T]$ satisfying

$$
\|h\|_{|\mathcal{H}|}^{2}=\int_{0}^{T} \int_{0}^{T}|h(t)||h(s)| \varphi(t, s) d s d t<\infty
$$

It is not difficult to show that $|\mathcal{H}|$ is a Banach space with the norm $\|\cdot\|_{|\mathcal{H}|}^{2}$.

In addition, we denote by $D_{t}^{H, k}$ the iteration of the derivative operator for any integer $k \geq 1$. The Sobolev space $\mathbb{D}^{k, p}$ is the closure of $\mathcal{S}$ with respect to the norm, for any $p \geq 1$ ( $\otimes$ denotes the tensor product),

$$
\|F\|_{k, p}^{p}=\mathbb{E}|F|^{p}+\mathbb{E} \sum_{j=1}^{k}\left\|D_{t}^{H, j} F\right\|_{\mathcal{H} \otimes j}^{p}
$$

Similarly, for a Hilbert space $U$, we denote by $\mathbb{D}^{k, p}(U)$ the corresponding Sobolev space of $U$-valued random variables. For any $p>0$ we denote by $\mathbb{D}^{1, p}(|\mathcal{H}|)$ the subspace of $\mathbb{D}^{1, p}(\mathcal{H})$ formed by the elements $h$ such that $h \in|\mathcal{H}|$.

Biagini et al. [14], Alos, Mazet and Nualart [24], Hu and Øksendal [9] have given more details as regards the $\mathrm{fBm}$.

Lemma 1 Let $u(t)$ be a stochastic process in the space $\mathbb{D}^{1,2}(|\mathcal{H}|)$, satisfying

$$
\int_{0}^{T} \int_{0}^{T}\left|D_{s}^{H} u(t)\right||t-s|^{2 H-2} d s d t<\infty
$$

then the symmetric integral coincides with the forward and backward integrals (P159,[14]).

Definition 2 The space $\mathcal{L}_{\varphi}[0, T]$ of integrands is defined as the family of stochastic processes $u(t)$ on $[0, T]$, such that $\mathbb{E}\|u(t)\|_{\varphi}^{2}<\infty, u(t)$ is $\varphi$-differentiable, the trace of $D_{s}^{\varphi} u(t)$ exists, $0 \leq s \leq T, 0 \leq t \leq T$, and

$$
\mathbb{E} \int_{0}^{T} \int_{0}^{T}\left[D_{t}^{\varphi} u(s)\right]^{2} d s d t<\infty,
$$


and for each sequence of partitions $\left(\pi_{n}, n \in \mathbb{N}\right)$ such that $\left|\pi_{n}\right| \rightarrow 0$ as $n \rightarrow \infty$,

$$
\sum_{i=0}^{n-1} \mathbb{E}\left[\int_{t_{i}^{(n)}}^{t_{i+1}^{(n)}} \int_{t_{j}^{(n)}}^{t_{j+1}^{(n)}}\left|D_{s}^{\varphi} u^{\pi}\left(t_{i}^{(n)}\right) D_{t}^{\varphi} u^{\pi}\left(t_{j}^{(n)}\right)-D_{s}^{\varphi} u(t) D_{t}^{\varphi} u(s)\right| d s d t\right]
$$

and

$$
\mathbb{E}\left[\left\|u^{\pi}-u\right\|_{\varphi}^{2}\right]
$$

tend to 0 as $n \rightarrow \infty$, where $\pi_{n}=t_{0}^{(n)}<t_{1}^{(n)}<\cdots<t_{n-1}^{(n)}<t_{n}^{(n)}=T$.

Lemma 3 Let $B^{H}(t)$ be a fBm with $\frac{1}{2}<H<1$, and $u(t)$ be a stochastic process in $\mathbb{D}^{1,2}(|\mathcal{H}|) \cap$ $\mathcal{L}_{\varphi}[0, T]$, then for every $T<\infty$,

$$
\mathbb{E}\left[\int_{0}^{T} u(s) d^{\circ} B^{H}(s)\right]^{2} \leq 2 H T^{2 H-1} \mathbb{E}\left[\int_{0}^{T}|u(s)|^{2} d s\right]+4 T \mathbb{E} \int_{0}^{T}\left[D_{s}^{\varphi} u(s)\right]^{2} d s .
$$

The detailed proof of Lemma 3 can be found in the authors' previous work [28-30].

In this paper, we always assume the following non-Lipschitz condition, which was proposed by Yamada and Watanabe [22], is satisfied.

\section{Hypothesis 4}

(1) There exists a function $\kappa(q)>0, q>0, \kappa(0)=0$ such that $\kappa(q)$ is a continuous non-decreasing, concave function and $\int_{0+} \frac{d q}{\kappa(q)}=+\infty$,

(2) $b(t, 0), \sigma(t, 0)$ are locally integral with respect to $t$,

(3) Furthermore, $\forall t \in[0, T], b(t, \cdot), \sigma(t, \cdot) \in \mathcal{L}_{\varphi}[0, T] \cap \mathbb{D}^{1,2}(|\mathcal{H}|)$, we have

$$
\begin{aligned}
& \mathbb{E}|b(t, X)-b(t, Y)|^{2}+\mathbb{E}|\sigma(t, X)-\sigma(t, Y)|^{2} \\
& \quad+\mathbb{E}\left|D_{t}^{\varphi}(\sigma(t, X)-\sigma(t, Y))\right|^{2} \leq \kappa\left(\mathbb{E}|X-Y|^{2}\right) .
\end{aligned}
$$

The above-mentioned Hypothesis 4 is the so-called non-Lipschitz condition. The nonLipschitz condition has a variety of forms [31-34]. Here, we consider one kind of them. In particular, we see clearly that if we let $\kappa(q)=K^{\prime} q$, then the non-Lipschitz condition reduces to the Lipschitz condition. In other words, the non-Lipschitz condition is weaker than the Lipschitz condition.

Now, we give some concrete examples of the function $\kappa$. Let $K^{\prime}>0$ and let $\left.\mu \in\right] 0,1[$ be sufficiently small. Define

$$
\begin{aligned}
\kappa_{1}(x) & =K^{\prime} x, \quad x \geq 0, \\
\kappa_{2}(x) & = \begin{cases}x \log \left(x^{-1}\right), & 0 \leq x \leq \mu, \\
\mu \log \left(\mu^{-1}\right)+\kappa_{2}^{\prime}(\mu-)(x-\mu), & x>\mu,\end{cases} \\
\kappa_{3}(x) & = \begin{cases}x \log \left(x^{-1}\right) \log \log \left(x^{-1}\right), & 0 \leq x \leq \mu, \\
\mu \log \left(\mu^{-1}\right) \log \log \left(\mu^{-1}\right)+\kappa_{3}^{\prime}(\mu-)(x-\mu), & x>\mu,\end{cases}
\end{aligned}
$$


where $\kappa^{\prime}$ denotes the derivative of the function $\kappa$. They are all concave and non-decreasing functions satisfying $\int_{0+} \frac{1}{\kappa_{i}(x)} d x=\infty(i=1,2,3)$.

\section{The main theorems}

In this section, using an iteration of the Picard type, we will discuss the solutions for non-Lipschitz SDEs with fBm. Let $X_{0}(t) \equiv \xi$ be a random variable with $\mathbb{E}|\xi|^{2}<$ $+\infty$, and construct an approximate sequence of stochastic process $\left\{X_{k}(t)\right\}_{k \geq 1}$ as follows:

$$
X_{k}(t)=\xi+\int_{0}^{t} b\left(s, X_{k-1}(s)\right) d s+\int_{0}^{t} \sigma\left(s, X_{k-1}(s)\right) d^{\circ} B^{H}(s), \quad k=1,2, \ldots
$$

Hereafter, we assume that $1 \leq T<+\infty$ without losing generality.

First, we given the following four key lemmas. The proofs for Lemma 5 and Lemma 6 will be presented in the Appendix.

Lemma 5 There exists a positive number $K, \forall b(t, \cdot), \sigma(t, \cdot) \in \mathcal{L}_{\varphi}[0, T] \cap \mathbb{D}^{1,2}(|\mathcal{H}|), t \in$ $[0, T]$, and we have

$$
\mathbb{E}|b(t, X)|^{2}+\mathbb{E}|\sigma(t, X)|^{2}+\mathbb{E}\left|D_{t}^{\varphi} \sigma(t, X)\right|^{2} \leq K\left(1+\mathbb{E}|X|^{2}\right) .
$$

Lemma 6 Under the conclusion of Lemma 5, one can get

$$
\mathbb{E}\left|X_{k}(t)\right|^{2} \leq C_{1}, \quad k=1,2, \ldots, t \in[0, T],
$$

where $C_{1}=3\left(1+\mathbb{E}|\xi|^{2}\right) \exp \left(12 K T^{2}\right)$.

Lemma 7 If $b(t, X)$ and $\sigma(t, X)$ satisfy the Hypothesis 4 , then for $t \in[0, T], n \geq 1, k \geq 1$, we have

$$
\mathbb{E}\left|X_{n+k}(s)-X_{n}(s)\right|^{2} \leq C_{2} \int_{0}^{t} \kappa\left(\mathbb{E}\left|X_{n+k-1}(s)-X_{n-1}(s)\right|^{2}\right) d s
$$

and

$$
\sup _{0 \leq s \leq t} \mathbb{E}\left|X_{n+k}(s)-X_{n}(s)\right|^{2} \leq C_{3} t
$$

where $C_{2}=8 T$ and $C_{3}$ is a constant.

Proof For $0 \leq s \leq t$, we show that

$$
\begin{aligned}
\mathbb{E}\left|X_{n+k}(s)-X_{n}(s)\right|^{2} \\
\leq 2 \mathbb{E}\left|\int_{0}^{s}\left(b\left(s_{1}, X_{n+k-1}\left(s_{1}\right)\right)-b\left(s_{1}, X_{n-1}\left(s_{1}\right)\right)\right) d s_{1}\right|^{2} \\
\quad+2 \mathbb{E}\left|\int_{0}^{s}\left(\sigma\left(s_{1}, X_{n+k-1}\left(s_{1}\right)\right)-\sigma\left(s_{1}, X_{n-1}\left(s_{1}\right)\right)\right) d^{\circ} B^{H}\left(s_{1}\right)\right|^{2} \\
\leq 8 T \mathbb{E} \int_{0}^{t}\left[\mid b\left(s_{1}, X_{n+k-1}\left(s_{1}\right)\right)-b\left(s_{1},\left.X_{n-1}\left(s_{1}\right)\right|^{2}\right.\right.
\end{aligned}
$$




$$
\begin{aligned}
& +\mid \sigma\left(s_{1}, X_{n+k-1}\left(s_{1}\right)\right)-\sigma\left(s_{1},\left.X_{n-1}\left(s_{1}\right)\right|^{2}\right. \\
& \left.+\left|D_{s_{1}}^{\varphi}\left(\sigma\left(s_{1}, X_{n+k-1}\left(s_{1}\right)\right)-\sigma\left(s_{1}, X_{n-1}\left(s_{1}\right)\right)\right)\right|^{2}\right] d s_{1} \\
\leq & C_{2} \int_{0}^{t} \kappa\left(\mathbb{E}\left|X_{n+k-1}(s)-X_{n-1}(s)\right|^{2}\right) d s .
\end{aligned}
$$

Then it is easy to verify

$$
\begin{aligned}
\sup _{0 \leq s \leq t} \mathbb{E}\left|X_{n+k}(s)-X_{n}(s)\right|^{2} & \leq C_{2} \int_{0}^{t} \kappa\left(\mathbb{E}\left|X_{n+k-1}(s)-X_{n-1}(s)\right|^{2}\right) d s \\
& \leq C_{2} \int_{0}^{t} \kappa\left(4 C_{1}\right) d s \leq C_{3} t .
\end{aligned}
$$

This completes the proof of Lemma 7.

Now, choose $0<T_{1} \leq T$, such that $t \in\left[0, T_{1}\right]$, for $\kappa_{1}\left(C_{3} t\right) \leq C_{3}, \kappa_{1}(q)=C_{2} \kappa(q)$ holds. We should note that in the following part, we first of all prove the following main theorem, Theorem 9, in the time interval $\left[0, T_{1}\right]$, then we extend the result in the whole interval $[0, T]$. Fix $k \geq 1$ arbitrarily and define two sequences of functions $\left\{\phi_{n}(t)\right\}_{n=1,2, \ldots}$ and $\left\{\tilde{\phi}_{n, k}(t)\right\}_{n=1,2, \ldots}$, where

$$
\begin{aligned}
& \phi_{1}(t)=C_{3} t \\
& \phi_{n+1}(t)=\int_{0}^{t} \kappa_{1}\left(\phi_{n}(s)\right) d s, \\
& \tilde{\phi}_{n, k}(t)=\sup _{0 \leq s \leq t} \mathbb{E}\left|X_{n+k}(s)-X_{n}(s)\right|^{2}, \quad n=1,2, \ldots
\end{aligned}
$$

Lemma 8 Under the Hypothesis 4,

$$
0 \leq \tilde{\phi}_{n, k}(t) \leq \phi_{n}(t) \leq \phi_{n-1}(t) \leq \cdots \leq \phi_{1}(t), \quad t \in\left[0, T_{1}\right],
$$

for all positive integer $n$.

Proof By Lemma 7, we have

$$
\tilde{\phi}_{1, k}(t)=\sup _{0 \leq s \leq t} \mathbb{E}\left|X_{1+k}(s)-X_{1}(s)\right|^{2} \leq C_{3} t=\phi_{1}(t), \quad t \in\left[0, T_{1}\right] .
$$

Then, since $\kappa_{1}(q)=C_{2} \kappa(q), \kappa(q)$ is a concave function and

$$
\mathbb{E}\left|X_{k+1}(s)-X_{1}(s)\right|^{2} \leq \sup _{0 \leq s \leq t} \mathbb{E}\left|X_{k+1}(s)-X_{1}(s)\right|^{2}=\tilde{\phi}_{1, k}(t), \quad 0 \leq s \leq t,
$$

it is easy to verify

$$
\begin{aligned}
\tilde{\phi}_{2, k}(t) & =\sup _{0 \leq s \leq t} \mathbb{E}\left|X_{2+k}(s)-X_{2}(s)\right|^{2} \\
& \leq C_{2} \int_{0}^{t} \kappa\left(\mathbb{E}\left|X_{k+1}(s)-X_{1}(s)\right|^{2}\right) d s
\end{aligned}
$$




$$
\begin{aligned}
& \leq \int_{0}^{t} \kappa_{1}\left(\tilde{\phi}_{1, k}(s)\right) d s \leq \int_{0}^{t} \kappa_{1}\left(\phi_{1}(s)\right) d s \\
& =\phi_{2}(t)=\int_{0}^{t} \kappa_{1}\left(C_{3} s\right) d s \\
& \leq C_{3} t=\phi_{1}(t), \quad t \in\left[0, T_{1}\right] .
\end{aligned}
$$

That is to say, for $n=2$, we have

$$
\tilde{\phi}_{2, k}(t) \leq \phi_{2}(t) \leq \phi_{1}(t), \quad t \in\left[0, T_{1}\right]
$$

Next, assume (3.4) for $n \geq 2$ and by the assumption for $n$

$$
\mathbb{E}\left|X_{n+k}(s)-X_{n}(s)\right|^{2} \leq \sup _{0 \leq s \leq t} \mathbb{E}\left|X_{n+k}(s)-X_{n}(s)\right|^{2}=\tilde{\phi}_{n, k}(t) \leq \phi_{n}(t)
$$

it is easy to verify for $n+1$

$$
\begin{aligned}
\tilde{\phi}_{n+1, k}(t) & =\sup _{0 \leq s \leq t} \mathbb{E}\left|X_{n+k+1}(s)-X_{n+1}(s)\right|^{2} \\
& \leq \int_{0}^{t} \kappa_{1}\left(\mathbb{E}\left|X_{n+k}(s)-X_{n}(s)\right|^{2}\right) d s \\
& \leq \int_{0}^{t} \kappa_{1}\left(\tilde{\phi}_{n, k}(s)\right) d s \\
& \leq \int_{0}^{t} \kappa_{1}\left(\phi_{n}(s)\right) d s=\phi_{n+1}(t) \\
& \leq \int_{0}^{t} \kappa_{1}\left(\phi_{n-1}(s)\right) d s=\phi_{n}(t), \quad t \in\left[0, T_{1}\right] .
\end{aligned}
$$

This completes the proof of Lemma 8.

Theorem 9 Under the Hypothesis 4, then

$$
\lim _{n, i \rightarrow \infty} \sup _{0 \leq t \leq T} \mathbb{E}\left|X_{n}(t)-X_{i}(t)\right|^{2}=0
$$

By Theorem 9, we say that $\left\{X_{k}(\cdot)\right\}_{k \geq 1}$ is a Cauchy sequence and define its limit as $X(\cdot)$. Then letting $k \rightarrow \infty$ in (3.1), we finally see that the solutions to (1.1) exist.

Proof Step 1: In this step we shall show

$$
\lim _{n, i \rightarrow \infty} \sup _{0 \leq t \leq T_{1}} \mathbb{E}\left|X_{n}(t)-X_{i}(t)\right|^{2}=0
$$

By Lemma 8, we know $\phi_{n}(t)$ decreases monotonically when $n \rightarrow \infty$ and $\phi_{n}(t)$ is nonnegative function on $t \in\left[0, T_{1}\right]$. Therefore, we can define the limit function $\phi(t)$ by $\phi_{n}(t) \downarrow$ $\phi(t)$. It is easy to verify that $\phi(0)=0$ and $\phi(t)$ is a continuous function on $t \in\left[0, T_{1}\right]$ [35]. According to the definition of $\phi_{n}(t)$ and $\phi(t)$, we obtain

$$
\phi(t)=\lim _{n \rightarrow \infty} \phi_{n+1}(t)=\lim _{n \rightarrow \infty} \int_{0}^{t} \kappa_{1}\left(\phi_{n}(s)\right) d s=\int_{0}^{t} \kappa_{1}(\phi(s)) d s, \quad t \in\left[0, T_{1}\right] .
$$


Since $\phi(0)=0$ and

$$
\int_{0+} \frac{d q}{\kappa_{1}(q)}=\frac{1}{C_{2}} \int_{0+} \frac{d q}{\kappa(q)}=+\infty
$$

(3.5) implies $\phi(t) \equiv 0, t \in\left[0, T_{1}\right]$.

Therefore we obtain

$$
0 \leq \lim _{k, n \rightarrow \infty} \sup _{0 \leq t \leq T_{1}} \mathbb{E}\left|X_{n+k}(t)-X_{n}(t)\right|^{2}=\lim _{k, n \rightarrow \infty} \tilde{\phi}_{n, k}\left(T_{1}\right) \leq \lim _{n \rightarrow \infty} \phi_{n}\left(T_{1}\right)=0
$$

namely,

$$
\lim _{n, i \rightarrow \infty} \sup _{0 \leq t \leq T_{1}} \mathbb{E}\left|X_{n}(t)-X_{i}(t)\right|^{2}=0
$$

Step 2: Define

$$
T_{2}=\sup \left\{\tilde{T}: \tilde{T} \in[0, T] \text { and } \lim _{n, i \rightarrow \infty} \sup _{0 \leq t \leq \tilde{T}} \mathbb{E}\left|X_{n}(t)-X_{i}(t)\right|^{2}=0\right\}
$$

Immediately, we can observe $0<T_{1} \leq T_{2} \leq T$. Now, we shall show

$$
\lim _{n, i \rightarrow \infty} \sup _{0 \leq t \leq T_{2}} \mathbb{E}\left|X_{n}(t)-X_{i}(t)\right|^{2}=0
$$

Let $\varepsilon>0$ be an arbitrary positive number. Choose $S_{0}$ so that $0<S_{0}<\min \left(T_{2}, 1\right)$. And

$$
C_{4} S_{0}<\frac{\varepsilon}{10}
$$

where $C_{4}=8 K\left(1+K_{1}\left(1+\mathbb{E}|\xi|^{2}\right)\right) S_{0}$.

From the definition of $T_{2}$, we have

$$
\lim _{n, i \rightarrow \infty} \sup _{0 \leq t \leq T_{2}-S_{0}} \mathbb{E}\left|X_{n}(t)-X_{i}(t)\right|^{2}=0 .
$$

Then, for large enough $N$, we observe

$$
\sup _{0 \leq t \leq T_{2}-S_{0}} \mathbb{E}\left|X_{n}(t)-X_{i}(t)\right|^{2}<\frac{\varepsilon}{10}, \quad n, i \geq N .
$$

On the other hand, one can get

$$
\begin{aligned}
\sup _{T_{2}-S_{0} \leq t \leq T_{2}} \mathbb{E}\left|X_{n}(t)-X_{i}(t)\right|^{2} \leq & 3 \sup _{T_{2}-S_{0} \leq t \leq T_{2}} \mathbb{E}\left|X_{n}(t)-X_{n}\left(T_{2}-S_{0}\right)\right|^{2} \\
& +3 \mathbb{E}\left|X_{n}\left(T_{2}-S_{0}\right)-X_{i}\left(T_{2}-S_{0}\right)\right|^{2} \\
& +3 \sup _{T_{2}-S_{0} \leq t \leq T_{2}} \mathbb{E}\left|X_{i}\left(T_{2}-S_{0}\right)-X_{i}(t)\right|^{2} \\
= & 3 I_{1}+3 I_{2}+3 I_{3} .
\end{aligned}
$$


Now, using Lemma 3, we obtain

$$
\begin{aligned}
I_{1}= & \sup _{T_{2}-S_{0} \leq t \leq T_{2}} \mathbb{E}\left|X_{n}(t)-X_{n}\left(T_{2}-S_{0}\right)\right|^{2} \\
\leq & 2 S_{0} \mathbb{E} \int_{T_{2}-S_{0}}^{T_{2}}\left|b\left(s_{1}, X_{n-1}\left(s_{1}\right)\right)\right|^{2} d s_{1} \\
& +4 H S_{0}{ }^{2 H-1} \mathbb{E} \int_{T_{2}-S_{0}}^{T_{2}}\left|\sigma\left(s_{1}, X_{n-1}\left(s_{1}\right)\right)\right|^{2} d s_{1} \\
& +8 S_{0} \mathbb{E} \int_{T_{2}-S_{0}}^{T_{2}}\left|D_{s_{1}}^{\varphi} \sigma\left(s_{1}, X_{n-1}\left(s_{1}\right)\right)\right|^{2} d s_{1} \\
\leq & 8 S_{0} \int_{T_{2}-S_{0}}^{T_{2}} K\left(1+K_{1}\left(1+\mathbb{E}|\xi|^{2}\right)\right) d s_{1} \\
\leq & 8 S_{0}^{2} K\left(1+K_{1}\left(1+\mathbb{E}|\xi|^{2}\right)\right) .
\end{aligned}
$$

Therefore by (3.6) we have

$$
I_{1} \leq \frac{\varepsilon}{10}
$$

and

$$
I_{3} \leq \frac{\varepsilon}{10}
$$

Meanwhile, (3.7) implies

$$
I_{2}=\mathbb{E}\left|X_{n}\left(T_{2}-S_{0}\right)-X_{i}\left(T_{2}-S_{0}\right)\right|^{2}<\frac{\varepsilon}{10}, \quad n, i \geq N
$$

Now putting (3.7)-(3.11) together, we have

$$
\begin{aligned}
\sup _{0 \leq t \leq T_{2}} \mathbb{E}\left|X_{n}(t)-X_{i}(t)\right|^{2} \leq & \sup _{0 \leq t \leq T_{2}-S_{0}} \mathbb{E}\left|X_{n}(t)-X_{i}(t)\right|^{2} \\
& +\sup _{T_{2}-S_{0} \leq t \leq T_{2}} \mathbb{E}\left|X_{n}(t)-X_{i}(t)\right|^{2} \\
\leq & \frac{\varepsilon}{10}+3 I_{1}+3 I_{2}+3 I_{3}<\varepsilon .
\end{aligned}
$$

That is to say,

$$
\lim _{n, i \rightarrow \infty} \sup _{0 \leq t \leq T_{2}} \mathbb{E}\left|X_{n}(t)-X_{i}(t)\right|^{2}=0
$$

Step 3: Using the method of reduction to absurdity, we shall show $T_{2}=T$. Assume $T_{2}<T$, we can choose a sequence of numbers $\left\{a_{i}\right\}_{i=1,2, \ldots}$ so that $a_{i} \downarrow 0(i \rightarrow+\infty)$ and for $n>i \geq 1$,

$$
\sup _{0 \leq t \leq T_{2}} \mathbb{E}\left|X_{n}(t)-X_{i}(t)\right|^{2} \leq a_{i}
$$

We shall divide the step into several sub-steps. 
First, for $n>i \geq 1$, we shall show

$$
\sup _{T_{2} \leq s \leq T_{2}+t} \mathbb{E}\left|X_{n}(s)-X_{i}(s)\right|^{2} \leq 3 a_{i}+C_{5} t, \quad T_{2}+t \leq T,
$$

where $C_{5}=12 T K\left(1+K_{1}\left(1+\mathbb{E}|\xi|^{2}\right)\right)$.

To show this, set

$$
\begin{aligned}
& J_{1}^{(i)}=\mathbb{E}\left|X_{n}\left(T_{2}\right)-X_{i}\left(T_{2}\right)\right|^{2}, \\
& J_{2}^{(i)}(t)=\sup _{T_{2} \leq s \leq T_{2}+t} \mathbb{E}\left|\int_{T_{2}}^{s}\left(b\left(s_{1}, X_{n-1}\left(s_{1}\right)\right)-b\left(s_{1}, X_{i-1}\left(s_{1}\right)\right)\right) d s_{1}\right|^{2}, \\
& J_{3}^{(i)}(t)=\sup _{T_{2} \leq s \leq T_{2}+t} \mathbb{E}\left|\int_{T_{2}}^{s}\left(\sigma\left(s_{1}, X_{n-1}\left(s_{1}\right)\right)-\sigma\left(s_{1}, X_{i-1}\left(s_{1}\right)\right)\right) d^{\circ} B^{H}\left(s_{1}\right)\right|^{2} .
\end{aligned}
$$

Then (3.12) implies $J_{1}^{(i)} \leq a_{i}$ and

$$
\begin{aligned}
J_{2}^{i}(t)+J_{3}^{i}(t) \leq & 4 T \mathbb{E} \int_{T_{2}}^{T_{2}+t}\left[\left|b\left(s_{1}, X_{n-1}\left(s_{1}\right)\right)-b\left(s_{1}, X_{i-1}\left(s_{1}\right)\right)\right|^{2}\right. \\
& +\left|\sigma\left(s_{1}, X_{n-1}\left(s_{1}\right)\right)-\sigma\left(s_{1}, X_{i-1}\left(s_{1}\right)\right)\right|^{2} \\
& \left.+\left|D_{s_{1}}^{\varphi}\left(\sigma\left(s_{1}, X_{n-1}\left(s_{1}\right)\right)-\sigma\left(s_{1}, X_{i-1}\left(s_{1}\right)\right)\right)\right|^{2}\right] d s_{1} \\
\leq & 4 T K\left(1+K_{1}\left(1+\mathbb{E}|\xi|^{2}\right)\right) t .
\end{aligned}
$$

Therefore

$$
\begin{aligned}
\sup _{T_{2} \leq s \leq T_{2}+t} \mathbb{E}\left|X_{n}(s)-X_{i}(s)\right|^{2} & \leq 3 J_{1}^{(i)}+3 J_{2}^{(i)}(t)+3 J_{3}^{(i)}(t) \\
& \leq 3 a_{i}+C_{5} t, \quad T_{2}+t \leq T .
\end{aligned}
$$

Next, we shall show an assertion which is analogous to Lemma 8. To state the assertion, we need to introduce several notations.

Choose a positive number $0<\eta \leq T-T_{2}$ and a positive integer $j \geq 1$, so that

$$
C_{6} \kappa\left(3 a_{j}+C_{5} t\right) \leq C_{5}, \quad t \in[0, \eta], \kappa_{2}(q)=C_{6} \kappa(q)
$$

where $C_{6}=12 T$.

Introduce the sequence of functions $\left\{\psi_{k}(t)\right\}_{k=1,2, \ldots}, t \in[0, \eta]$, defined by

$$
\begin{aligned}
& \psi_{1}(t)=3 a_{j}+C_{5} t \\
& \psi_{k+1}(t)=3 a_{j+k}+\int_{0}^{t} \kappa_{2}\left(\psi_{k}(s)\right) d s, \\
& \tilde{\psi}_{k, n}(t)=\sup _{T_{2} \leq s \leq T_{2}+t} \mathbb{E}\left|X_{n+k}(s)-X_{j+k}(s)\right|^{2} .
\end{aligned}
$$

Now, the assertion to be proved is the following:

$$
\tilde{\psi}_{k, n}(t) \leq \psi_{k}(t) \leq \psi_{k-1}(t) \leq \cdots \leq \psi_{1}(t), \quad t \in[0, \eta]
$$

for all positive integer $k$. 
Noticing that $\kappa_{2}(q)$ is a non-decreasing, concave function, and (3.13) holds, from this for $k=1$, we work out

$$
\begin{aligned}
\tilde{\psi}_{1, n}(t)= & \sup _{T_{2} \leq s \leq T_{2}+t} \mathbb{E}\left|X_{n+1}(s)-X_{j+1}(s)\right|^{2} \\
\leq & 3 a_{j+1}+C_{6} \mathbb{E} \int_{T_{2}}^{T_{2}+t}\left[\left|b\left(s_{1}, X_{n}\left(s_{1}\right)\right)-b\left(s_{1}, X_{j}\left(s_{1}\right)\right)\right|^{2}\right. \\
& +\left|\sigma\left(s_{1}, X_{n}\left(s_{1}\right)\right)-\sigma\left(s_{1}, X_{j}\left(s_{1}\right)\right)\right|^{2} \\
& \left.+\left|D_{s_{1}}^{\varphi}\left(\sigma\left(s_{1}, X_{n}\left(s_{1}\right)\right)-\sigma\left(s_{1}, X_{j}\left(s_{1}\right)\right)\right)\right|^{2}\right] d s_{1} \\
\leq & 3 a_{j+1}+\int_{T_{2}}^{T_{2}+t} \kappa_{2}\left(\mathbb{E}\left|X_{n}\left(s_{1}\right)-X_{j}\left(s_{1}\right)\right|^{2}\right) d s_{1} \\
\leq & 3 a_{j}+\int_{T_{2}}^{T_{2}+t} \kappa_{2}\left(3 a_{j}+C_{5} s_{1}\right) d s_{1} \leq \psi_{1}(t), \quad t \in[0, \eta] .
\end{aligned}
$$

On the other hand, using (3.14) we arrive at

$$
\begin{aligned}
\tilde{\psi}_{2, n}(t) & \leq \sup _{T_{2} \leq s \leq T_{2}+t} \mathbb{E}\left|X_{n+2}(s)-X_{j+2}(s)\right|^{2} \\
& \leq 3 a_{j+2}+C_{6} \int_{T_{2}}^{T_{2}+t} \kappa\left(\mathbb{E}\left|X_{n+1}\left(s_{1}\right)-X_{j+1}\left(s_{1}\right)\right|^{2}\right) d s_{1} \\
& \leq 3 a_{j+2}+\int_{T_{2}}^{T_{2}+t} \kappa_{2}\left(\tilde{\psi}_{1, n}(t)\right) d s_{1} \\
& \leq 3 a_{j+1}+\int_{T_{2}}^{T_{2}+t} \kappa_{2}\left(\psi_{1}(t)\right) d s_{1}=\psi_{2}(t) \\
& \leq 3 a_{j}+C_{5} t=\psi_{1}(t), \quad t \in[0, \eta] .
\end{aligned}
$$

Then we have proved

$$
\tilde{\psi}_{2, n}(t) \leq \psi_{2}(t) \leq \psi_{1}(t)
$$

Now assume that the assertion holds for $k \geq 2$. Then, by an analogous argument, one can obtain

$$
\begin{aligned}
\tilde{\psi}_{k+1, n}(t) & \leq 3 a_{j+k+1}+\int_{T_{2}}^{T_{2}+t} \kappa_{2}\left(\mathbb{E}\left|X_{n+k}\left(s_{1}\right)-X_{j+k}\left(s_{1}\right)\right|^{2}\right) d s_{1} \\
& \leq 3 a_{j+k+1}+\int_{T_{2}}^{T_{2}+t} \kappa_{2}\left(\tilde{\psi}_{k, n}\left(s_{1}\right)\right) d s_{1} \\
& \leq 3 a_{j+k}+\int_{T_{2}}^{T_{2}+t} \kappa_{2}\left(\psi_{k}\left(s_{1}\right)\right) d s_{1}=\psi_{k+1}(t) \\
& \leq 3 a_{j+k-1}+\int_{T_{2}}^{T_{2}+t} \kappa_{2}\left(\psi_{k-1}\left(s_{1}\right)\right) d s_{1} \\
& =\psi_{k}(t), \quad t \in[0, \eta] .
\end{aligned}
$$


Therefore, we obtain (3.15) for all $k$. In terms of (3.15), we can define the function $\psi(t)$ by $\psi_{k}(t) \downarrow \psi(t)(k \rightarrow \infty)$. We observe that

$$
\begin{aligned}
\psi(0) & =\lim _{k \rightarrow \infty} \psi_{k+1}(0) \\
& =\lim _{k \rightarrow \infty} a_{j+k}=0 .
\end{aligned}
$$

It is easy to verify that $\psi(t)$ is a continuous function on $[0, \eta]$. Now by the definition of $\psi_{k+1}(t)$ and $\psi(t)$, we have

$$
\begin{aligned}
\psi(t) & =\lim _{k \rightarrow \infty} \psi_{k+1}(t) \\
& =\lim _{k \rightarrow \infty}\left[3 a_{j+k}+\int_{0}^{t} \kappa_{2}\left(\psi_{k}(s)\right) d s\right] \\
& =\int_{0}^{t} \kappa_{2}(\psi(s)) d s .
\end{aligned}
$$

Since $\psi(0)=0$ and

$$
\int_{0+} \frac{d q}{\kappa_{2}(q)}=\frac{1}{C_{6}} \int_{0+} \frac{d q}{\kappa(q)}=+\infty
$$

(3.16) implies $\psi(t)=0, t \in[0, \eta]$.

Therefore, we obtain

$$
\begin{aligned}
\lim _{k \rightarrow \infty} \tilde{\psi}_{k, n}(t)= & \lim _{k \rightarrow \infty} \sup _{0 \leq s \leq T_{2}+t} \mathbb{E}\left|X_{n+k}(s)-X_{j+k}(s)\right|^{2} \\
\leq & \lim _{k \rightarrow \infty} \sup _{0 \leq s \leq T_{2}} \mathbb{E}\left|X_{n+k}(s)-X_{j+k}(s)\right|^{2} \\
& +\lim _{k \rightarrow \infty} \sup _{T_{2} \leq s \leq T_{2}+\eta} \mathbb{E}\left|X_{n+k}(s)-X_{j+k}(s)\right|^{2} \\
\leq & \lim _{k \rightarrow \infty} \psi_{k}(\eta)=\psi(\eta)=0,
\end{aligned}
$$

namely

$$
\lim _{n, i \rightarrow \infty} \sup _{0 \leq t \leq T_{2}+\eta} \mathbb{E}\left|X_{n}(t)-X_{i}(t)\right|^{2}=0
$$

But this conclusion is contradictory to the definition of $T_{2}$. In other words, we have already shown that

$$
\lim _{n, i \rightarrow \infty} \sup _{0 \leq t \leq T} \mathbb{E}\left|X_{n}(t)-X_{i}(t)\right|^{2}=0
$$

The proof of the existence of solutions of SDEs (1.1) is complete.

Theorem 10 Under the Hypothesis 4, the path-wise uniqueness holds for (1.1), $t \in[0, T]$. 
Proof Let $X(t)$ and $\tilde{X}(t)$ be two solutions of (1.1) on the same probability space and $X(0)=$ $\tilde{X}(0)$. We observe

$$
\begin{aligned}
& \mathbb{E}|X(t)-\tilde{X}(t)|^{2} \\
&=\mathbb{E}\left|\int_{0}^{t}(b(s, X(s))-b(s, \tilde{X}(s))) d s+\int_{0}^{t}\left(\sigma\left(s_{1}, X(s)\right)-\sigma(s, \tilde{X}(s))\right) d^{\circ} B^{H}(s)\right|^{2} \\
& \leq 2 \mathbb{E}\left|\int_{0}^{t}(b(s, X(s))-b(s, \tilde{X}(s))) d s\right|^{2}+2 \mathbb{E}\left|\int_{0}^{t}(\sigma(s, X(s))-\sigma(s, \tilde{X}(s))) d^{\circ} B^{H}(s)\right|^{2} \\
& \leq 8 T \mathbb{E} \int_{0}^{t}\left(|b(s, X(s))-b(s, \tilde{X}(s))|^{2}+|\sigma(s, X(s))-\sigma(s, \tilde{X}(s))|^{2}\right. \\
& \quad\left.\left|D_{s}^{\varphi}(\sigma(s, X(s))-\sigma(s, \tilde{X}(s)))\right|^{2}\right) d s .
\end{aligned}
$$

Combining the above inequalities and the Hypothesis 4, one has

$$
\mathbb{E}|X(t)-\tilde{X}(t)|^{2} \leq 8 T \int_{0}^{t} \kappa\left(\mathbb{E}|X(s)-\tilde{X}(s)|^{2}\right) d s
$$

Then, noticing that $\int_{0+} \frac{d q}{\kappa(q)}=+\infty$, the above inequality (3.17) implies

$$
\mathbb{E}|X(t)-\tilde{X}(t)|^{2}=0, \quad t \in[0, T]
$$

Since $T$ is an arbitrary positive number, we obtain from this $X(t) \equiv \tilde{X}(t)$, for all $0 \leq t \leq T$. Thus the path-wise uniqueness holds for (1.1).

\section{Appendix}

Proof of Lemma 5 Since $\kappa(q)$ is a concave and non-negative function, we can choose two positive constants $a>0$ and $b>0$, so that

$$
\kappa(q) \leq a+b q, \quad q \geq 0
$$

then, by (2.1), we get

$$
\begin{aligned}
\mathbb{E}|\sigma(t, X)|^{2}+\mathbb{E}|b(t, X)|^{2}+\mathbb{E}\left|D_{t}^{\varphi} \sigma(t, X)\right|^{2} \\
\leq 2 \mathbb{E}\left(|\sigma(t, 0)|^{2}+|b(t, 0)|^{2}+\left|D_{t}^{\varphi} \sigma(t, 0)\right|^{2}\right)+2 \mathbb{E}|\sigma(t, X)-\sigma(t, 0)|^{2} \\
\quad+2 \mathbb{E}|b(t, X)-b(t, 0)|^{2}+2 \mathbb{E}\left|D_{t}^{\varphi}(\sigma(t, X)-\sigma(t, 0))\right|^{2} \\
\leq 2 \sup _{0 \leq t \leq T} \mathbb{E}\left(|\sigma(t, 0)|^{2}+|b(t, 0)|^{2}+\left|D_{t}^{\varphi} \sigma(t, 0)\right|^{2}\right)+2 \kappa\left(\mathbb{E}|X|^{2}\right) \\
\leq K\left(1+\mathbb{E}|X|^{2}\right),
\end{aligned}
$$

where $K=\max \left[2 \sup _{0 \leq t \leq T} \mathbb{E}\left(|\sigma(t, 0)|^{2}+|b(t, 0)|^{2}+\left|D_{t}^{\varphi} \sigma(t, 0)\right|^{2}\right)+2 a, 2 b\right]<+\infty$ 
Proof of Lemma 6 Using mathematical induction, we first assume that

$$
\mathbb{E}\left|X_{k}(t)\right|^{2} \leq 3 \mathbb{E}|\xi|^{2} \sum_{l=0}^{k} \frac{(12 K T)^{l}}{l !} t^{l}+\sum_{l=1}^{k} \frac{(12 K T)^{l}}{l !} t^{l}
$$

holds, $t \in[0, T], k=1,2, \ldots$.

Clearly, by Lemma 3 and Lemma 5, we arrive at

$$
\begin{aligned}
\mathbb{E}\left|X_{1}(t)\right|^{2} & \leq 3 \mathbb{E}|\xi|^{2}+3 \mathbb{E}\left|\int_{0}^{t} b\left(s, X_{0}(s)\right) d s\right|^{2}+3 \mathbb{E}\left|\int_{0}^{t} \sigma\left(s, X_{0}(s)\right) d^{\circ} B^{H}(s)\right|^{2} \\
& \leq 3 \mathbb{E}|\xi|^{2}+12 T \mathbb{E} \int_{0}^{t}\left(\left|b\left(s, X_{0}(s)\right)\right|^{2}+\left|\sigma\left(s, X_{0}(s)\right)\right|^{2}+\left|D_{s}^{\varphi} \sigma\left(s, X_{0}(s)\right)\right|^{2}\right) d s \\
& \leq 3 \mathbb{E}|\xi|^{2}+12 K T t\left(1+\mathbb{E}|\xi|^{2}\right) .
\end{aligned}
$$

Now, assume that (A.1) holds for $k$, then we have, for $k+1$,

$$
\begin{aligned}
\mathbb{E}\left|X_{k+1}(t)\right|^{2} & \leq 3 \mathbb{E}|\xi|^{2}+3 \mathbb{E}\left|\int_{0}^{t} b\left(s, X_{k}(s)\right) d s\right|^{2}+3 \mathbb{E}\left|\int_{0}^{t} \sigma\left(s, X_{k}(s)\right) d^{\circ} B^{H}(s)\right|^{2} \\
& \leq 3 \mathbb{E}|\xi|^{2}+12 T \mathbb{E} \int_{0}^{t}\left(\left|b\left(s, X_{k}(s)\right)\right|^{2}+\left|\sigma\left(s, X_{k}(s)\right)\right|^{2}+\left|D_{s}^{\varphi} \sigma\left(s, X_{k}(s)\right)\right|^{2}\right) d s \\
& \leq 3 \mathbb{E}|\xi|^{2}+12 K T \int_{0}^{t}\left(1+\mathbb{E}\left|X_{k}(s)\right|^{2}\right) d s \\
& \leq 3 \mathbb{E}|\xi|^{2}+12 K T \int_{0}^{t}\left(1+3 \mathbb{E}|\xi|^{2} \sum_{l=0}^{k} \frac{(12 K T)^{l}}{l !} s^{l}+\sum_{l=1}^{k} \frac{(12 K T)^{l}}{l !} s^{l}\right) d s \\
& =3 \mathbb{E}|\xi|^{2}+12 K T t+3 \mathbb{E}|\xi|^{2} \sum_{l=1}^{k+1} \frac{(12 K T)^{l}}{l !} t^{l}+\sum_{l=2}^{k+1} \frac{(12 K T)^{l}}{l !} t^{l} \\
& =3 \mathbb{E}|\xi|^{2} \sum_{l=0}^{k+1} \frac{(12 K T)^{l}}{l !} t^{l}+\sum_{l=1}^{k+1} \frac{(12 K T)^{l}}{l !} t^{l} .
\end{aligned}
$$

Therefore, by induction, (A.1) holds for all $k$.

Now, we obtain the form $C_{1}=3\left(1+\mathbb{E}|\xi|^{2}\right) \exp \left(12 K T^{2}\right)$, then (A.2) implies (3.2).

Competing interests

The authors declare that they have no competing interests.

\section{Authors' contributions}

All authors contributed equally to the writing of this paper. All authors read and approved the final manuscript.

\section{Acknowledgements}

This work was supported by the NSF of China (11572247), the Fundamental Research Funds for the Central Universities and Innovation Foundation for Doctor Dissertation of Northwestern Polytechnical University. Authors would like to thank the referees for their helpful comments.

Received: 9 February 2016 Accepted: 30 June 2016 Published online: 23 July 2016

\section{References}

1. Øksendal, B: Stochastic Differential Equations. Springer, Berlin (2005)

2. Arnold, L: Stochastic Differential Equations, Theory and Applications. John Wiley and Sons, New York (1974)

3. Friedman, A: Stochastic Differential Equations and Applications. Dover Publications, New York (2006)

4. Gard, T: Introduction to Stochastic Differential Equations. Marcel Dekker, New York (1988) 
5. Hurst, H: Long-term storage capacity in reservoirs. Trans. Am. Soc. Civ. Eng. 116, 400-410 (1951)

6. Kolmogorov, A: Wienersche spiralen und einige andere interessante kurven im Hilbertschen raum. C. R. (Dokl.) Acad. Sci. URSS 26, 115-118 (1940)

7. Mandelbrot, B, Van Ness, J: Fractional Brownian motions, fractional noises and applications. SIAM Rev. 10(4), 422-427 (1968)

8. Chakravarti, N, Sebastian, K: Fractional Brownian motion models for polymers. Chem. Phys. Lett. 267, 9-13 (1997)

9. Hu, Y, Øksendal, B: Fractional white noise calculus and application to finance. Infin. Dimens. Anal. Quantum Probab. Relat. Top. 6, 1-32 (2003)

10. Scheffer, R, Maciel, F: The fractional Brownian motion as a model for an industrial airlift reactor. Chem. Eng. Sci. 56, 707-711 (2001)

11. Lyons, T: Differential equations driven by rough signals. Rev. Mat. Iberoam. 14, 215-310 (1998)

12. Nualart, D, Rascanu, A: Differential equations driven by fractional Brownian motion. Collect. Math. 53, 55-81 (2002)

13. Mishura, Y: Stochastic Calculus for Fractional Brownian Motion and Related Processes. Springer, Berlin (2008)

14. Biagini, $F, H u, Y, O k s e n d a l, B$, Zhang, T: Stochastic Calculus for Fractional Brownian Motion and Applications. Springer, London (2008)

15. Cox, J, Ingersoll, J, Ross, S: A theory of the term structure of interest rate. Econometrica 53, 385-407 (1985)

16. Taniguchi, T: Successive approximations to solutions of stochastic differential equations. J. Differ. Equ. 96, 152-169 (1992)

17. Kwok, Y: Pricing multi-asset options with an external barrier. Int. J. Theor. Appl. Finance 1, 523-541 (1998)

18. Watanabe, S, Yamada, T: On the uniqueness of solution of stochastic differential equations II. J. Math. Kyoto Univ. 11(3), 553-563 (1971)

19. Barlow, M: One dimensional stochastic differential equations with no strong solution. J. Lond. Math. Soc. 26, 335-347 (1982)

20. Yamada, T: On a comparison theorem for solutions of stochastic differential equations and its applications. J. Math. Kyoto Univ. 13(3), 497-512 (1973)

21. Yamada, T: On the successive approximation of solutions of stochastic differential equations. J. Math. Kyoto Univ. 21(3), 501-515 (1981)

22. Yamada, T, Watanabe, S: On the uniqueness of solutions of stochastic differential equations. J. Math. Kyoto Univ. 11, 155-167 (1971)

23. Carmona, P, Coutin, L, Montseny, G: Stochastic integration with respect to fractional Brownian motion. Ann. Inst. Henri Poincaré Probab. Stat. 39, 27-68 (2003)

24. Alòs, E, Mazet, O, Nualart, D: Stochastic calculus with respect to Gaussian process. Ann. Probab. 29, 766-801 (2001)

25. Duncan, T, Hu, Y, Pasik-Duncan, B: Stochastic calculus for fractional Brownian motion I: theory. SIAM J. Control Optim. 38, 582-612 (2000)

26. Alòs, E, Nualart, D: Stochastic integration with respect to the fractional Brownian motion. Stoch. Stoch. Rep. 75(3), 129-152 (2003)

27. Russo, F, Vallois, P: Forward, backward and symmetric stochastic integration. Probab. Theory Relat. Fields 97, 403-421 (1993)

28. $\mathrm{Xu}, \mathrm{Y}, \mathrm{Pei}, \mathrm{B}, \mathrm{Guo}, \mathrm{R}$ : Stochastic averaging for slow-fast dynamical systems with fractional Brownian motion. Discrete Contin. Dyn. Syst., Ser. B 20, 2257-2267 (2015)

29. $\mathrm{Xu}, \mathrm{Y}, \mathrm{Guo}, \mathrm{R}$, et al.: Stochastic averaging principle for dynamical systems with fractional Brownian motion. Discrete Contin. Dyn. Syst., Ser. B 19(4), 1197-1212 (2014)

30. $\mathrm{Xu}, \mathrm{Y}, \mathrm{Pei}, \mathrm{B}, \mathrm{Li}, \mathrm{Y}$ : An averaging principle for stochastic differential delay equations with fractional Brownian motion. Abstr. Appl. Anal. 2014, Article ID 479195 (2014)

31. Albeverio, S, Brzézniak, Z, Wu, J: Existence of global solutions and invariant measures for stochastic differential equations driven by Poisson type noise with non-Lipschitz coefficients. J. Math. Anal. Appl. 371, 309-322 (2010)

32. Taniguchi, T: The existence and uniqueness of energy solutions to local non-Lipschitz stochastic evolution equations. J. Math. Anal. Appl. 360, 245-253 (2009)

33. Barbu, D, Bocsan, G: Approximations to mild solutions of stochastic semilinear equations with non-Lipschitz coefficients. Czechoslov. Math. J. 52, 87-95 (2002)

34. $\mathrm{Xu}, \mathrm{Y}, \mathrm{Pei}, \mathrm{B}, \mathrm{Wu}$, J: Stochastic averaging principle for differential equations with non-Lipschitz coefficients driven by fractional Brownian motion. Stoch. Dyn. (2016). doi:10.1142/S0219493717500137

35. $\mathrm{Xu}, \mathrm{Y}, \mathrm{Pei}, \mathrm{B}, \mathrm{Guo}, \mathrm{G}$ : Existence and stability of solutions to non-Lipschitz stochastic differential equations driven by Lévy noise. Appl. Math. Comput. 263, 398-409 (2015)

\section{Submit your manuscript to a SpringerOpen ${ }^{\circ}$ journal and benefit from:}

- Convenient online submission

Rigorous peer review

- Immediate publication on acceptance

- Open access: articles freely available online

- High visibility within the field

- Retaining the copyright to your article 